\title{
Structural basis for translational shutdown and immune evasion by the Nsp1 protein of SARS-CoV-2
}

\author{
Matthias Thoms ${ }^{1 *}$, Robert Buschauer ${ }^{1 *}$, Michael Ameismeier ${ }^{1 *}$, Lennart Koepke $^{2}$, Timo Denk', \\ Maximilian Hirschenberger ${ }^{2}$, Hanna Kratzat ${ }^{1}$, Manuel Hayn ${ }^{2}$, Timur Mackens-Kiani' ${ }^{1}$, Jingdong Cheng', \\ Jan H. Straub ${ }^{2}$, Christina M. Stürzel ${ }^{2}$, Thomas Fröhlich ${ }^{3}$, Otto Berninghausen', Thomas Becker ${ }^{1}$, \\ Frank Kirchhoff ${ }^{2}$, Konstantin M. J. Sparrer ${ }^{2}+$, Roland Beckmann ${ }^{1 \dagger}$
}

${ }^{1}$ Gene Center Munich, Department of Biochemistry, University of Munich, Munich, Germany. ${ }^{2}$ Institute of Molecular Virology, Ulm University Medical Center, Ulm, Germany. ${ }^{3}$ Laboratory of Functional Genome Analysis, University of Munich, Munich, Germany.

*These authors contributed equally to this work. †Corresponding author. Email: beckmann@genzentrum.Imu.de (R.B.); konstantin.sparrer@uni-ulm.de (K.M.J.S.)

SARS-CoV-2 is the causative agent of the current COVID-19 pandemic. A major virulence factor of SARS$\mathrm{CoVs}$ is the nonstructural protein 1 (Nsp1) which suppresses host gene expression by ribosome association. Here, we show that Nsp1 from SARS-CoV-2 binds to the 40S ribosomal subunit, resulting in shutdown of mRNA translation both in vitro and in cells. Structural analysis by cryo-electron microscopy (cryo-EM) of in vitro reconstituted Nsp1-40S and various native Nsp1-40S and -80S complexes revealed that the Nsp1 C terminus binds to and obstructs the mRNA entry tunnel. Thereby, Nsp1 effectively blocks RIG-I-dependent innate immune responses that would otherwise facilitate clearance of the infection. Thus, the structural characterization of the inhibitory mechanism of Nsp1 may aid structure-based drug design against SARSCoV-2.

Coronaviruses (CoVs) are enveloped, single-stranded viruses with a positive-sense RNA genome, which infect a large variety of vertebrate animal species. Currently, seven $\mathrm{CoV}$ species from two genera (alpha and beta) are known human pathogens, four of which usually cause only mild respiratory diseases like common colds (1-5). Over the last two decades, however, three Betacoronaviruses (beta-CoVs) - the severe acute respiratory syndrome-coronavirus (SARS-CoV), the Middle East respiratory syndrome-coronavirus (MERS-CoV) and the novel severe acute respiratory syndrome-coronavirus 2 (SARS-CoV-2) - have emerged as the causative agents of epidemic and in the case of SARS-CoV-2 pandemic outbreaks of highly pathogenic respiratory diseases. COVID-19, the disease caused by SARS-CoV-2 has affected millions of people with a death toll amounting to hundreds of thousands worldwide ( 6 , 7).

Coronavirus particles contain a single, 5'-capped and 3'poly-adenylated RNA genome, which codes for two large overlapping open reading frames in gene 1 (ORFla and ORF1b), as well as a variety of structural and nonstructural proteins at the $3^{\prime}$ end $(8,9)$. Following host infection, precursor proteins ORFla and ORFlab are translated and subsequently proteolytically cleaved into functional proteins, most of which play roles during viral replication (10). Amongst them is the N-terminal nonstructural protein 1 (Nsp1). Despite differences in protein size and mode of action, Nsp1 proteins from alpha- and beta-CoVs display a similar biological function in suppressing host gene expression (11-14). SARS-
CoV Nsp1 induces a near-complete shutdown of host protein translation by a two-pronged strategy: first, it binds the small ribosomal subunit and stalls canonical mRNA translation at various stages during initiation $(15,16)$. Second, Nsp1 binding to the ribosome leads to endonucleolytic cleavage and subsequent degradation of host mRNAs. Notably, interactions between Nsp1 and a conserved region in the $5^{\prime}$ untranslated region (UTR) of viral mRNA prevent shutdown of viral protein expression through an unknown mechanism (17). Taken together, Nsp1 inhibits all cellular anti-viral defense mechanisms that depend on the expression of host factors, including the interferon response. This shutdown of the key parts of the innate immune system may facilitate efficient viral replication $(13,18)$ and immune evasion. Its central role in weakening the anti-viral immune response makes SARS-CoV Nsp1 a potential therapeutic target $(19,20)$. Here, we set out to characterize the interaction of Nsp1 of SARS-CoV-2 with the human translation machinery.

Nsp1 of SARS-CoV-2 shows $84 \%$ amino acid sequence identity with SARS-CoV, suggesting similar properties and biological functions (Fig. 1A). The C-terminal residues K164 and H165 in SARS-CoV are conserved in beta-CoVs and essential for $40 \mathrm{~S}$ interaction since mutations to alanine abolish $40 \mathrm{~S}$ binding and relieve translational inhibition (16). To confirm an analogous function of Nsp1 from SARS-CoV-2, we expressed and purified recombinant Nsp1 and the K164A/H165A mutant (Nsp1-mt) of both SARS-CoV and SARS-CoV-2 from E. coli, and tested their binding efficiency 
to purified human ribosomal subunits (Fig. 1B and fig. S1A). Nsp1 from both CoVs associated strongly with 40S subunits but not with $60 \mathrm{~S}$ subunits, whereas both Nsp1-mt constructs showed no binding (Fig. 1B). Thus, ribosome binding to the 40S subunit is preserved and residues K164 and H165 of Nsp1 from both SARS-CoVs are important for this ribosome interaction. To further verify this, we expressed wildtype or mutant Nsp1 constructs in human HEK293T cells and analyzed ribosome association by sucrose-gradient centrifugation. Consistent with the behavior in vitro, Nsp1 of CoV and CoV2 co-migrated with $40 \mathrm{~S}$ ribosomal subunits and $80 \mathrm{~S}$ ribosomes, but not with actively translating polyribosomes. In contrast, the mutant constructs barely penetrated the gradient, indicative of their loss of affinity for ribosomes (Fig. 1C). Notably, compared to the control the polysome profiles showed a shift from translating polyribosomes to $80 \mathrm{~S}$ monosomes in the presence of Nsp1, indicating global inhibition of translation. This effect was less pronounced for the two Nsp1mt constructs. Next, we performed in vitro translation assays of capped reporter mRNA in cell free translation extracts from human cells (HeLa S3) or rabbit reticulocytes in the presence of Nsp1 or Nsp1-mt. Probing for the translation products by Western blotting revealed a complete inhibition of translation by Nsp1 and only weak effects in the presence of Nsp1-mt constructs (Fig. 1D and fig. S1B). To test the inhibitory effect of Nsp1 on translation in cells, we expressed 3xFLAG-tagged Nsp1 of SARS-CoV-2 and SARS-CoV and their respective mutants in HEK293T cells and monitored translation of a co-transfected capped luciferase reporter mRNA. Consistent with the results of the in vitro assays, we observed a strong reduction of translation in presence of Nsp1 from SCoV-1 or -2 , but not of the respective Nsp1-mt constructs (Fig. 1E). This phenotype was confirmed for differently tagged (V5) and codon-optimized versions of SCoV-2 Nsp1 (fig. S1, C and D). Nsp7, which is derived from the same polyprotein precursor as Nsp1 had no effect on translation (fig. S1C). In summary, Nsp1 from both, SARS-CoV and SARS-CoV2 binds $40 \mathrm{~S}$ and $80 \mathrm{~S}$ ribosomes and disrupts cap-dependent translation. Moreover, the conserved $\mathrm{KH}$ motif close to the $\mathrm{C}$ terminus of Nsp1 is crucial for ribosome binding and translation inhibition.

To elucidate the molecular interaction of SARS-CoV-2 Nsp1 with human ribosomes, we reconstituted a complex from purified, recombinant Nsp1 and purified human 40S ribosomal subunits and determined its structure by cryo-EM at an average resolution of $2.6 \AA$ (Fig. 2, A and B, and figs. S2 and S3). In addition to the $40 \mathrm{~S}$ ribosomal subunit, we observed density corresponding to two $\alpha$-helices inside the ribosomal mRNA entry channel, which could be unambiguously identified as the C-terminal part of Nsp1 from SARS-CoV-2 (Fig. 2C). In proximity to the helical density, we observed undefined globular density between rRNA helix h16 and ribosomal proteins uS3 and uS10. The dimensions of this extra density roughly match the putative dimensions of the globular N-terminal domain of Nsp1 (Fig. 2, C and D), based on a structure of the highly similar N terminus of Nsp1 from SARS-CoV, previously determined by NMR (21). However, the resolution of this region in our cryo-EM density map was insufficient for unambiguous identification. The $\mathrm{C}$ terminus of Nsp1 is located close to the so called "latch" between rRNA helix h18 of the body and h34 of the head of the 40S subunit, which influences mRNA accommodation and movement during translation initiation $(22,23)$. When bound at this position, the Nsp1 $\mathrm{C}$ terminus blocks regular mRNA accommodation, thus providing an explanation for Nsp1 mediated host translation shutdown (Fig. 2D).

To characterize the ribosomal targets and the mode of interaction of Nsp1 in human cells, we expressed N-terminally 3xFLAG tagged Nsp1 in HEK293T cells and affinity purified associated native complexes for analysis by cryo-EM and mass spectrometry (Fig. 2E, figs. S2 and S3, and data S1). Structural analysis revealed $40 \mathrm{~S}$ and $80 \mathrm{~S}$ ribosomal complexes in nine compositionally different states (Fig. 2, F to N). Importantly, all of them displayed density for the Nsp1 C terminus in an identical position and conformation observed in the in vitro assembled complex, and all complexes lacked density corresponding to mRNA.

The Nsp1-bound 40S ribosomal complexes could be divided into three major populations. The first represents idle Nsp1-40S complexes (Fig. 2F), essentially resembling the in vitro reconstituted complex. The second population comprises unusual, pre-40S-like complexes (Fig. 2, G and H), in which the cytosolic ribosome biogenesis factor TSR1 is bound in two distinct conformations between the 40S head and body $(24,25)$. Notably, these complexes do not resemble any known on-pathway biogenesis intermediates. The third population represents eIF3-containing $43 \mathrm{~S}$ pre-initiation complexes (PICs), and could be further divided into PICs with and without eIF1A, eIF1 and a fully assembled eIF2-tRNA $\mathrm{i}_{\mathrm{i}}$-GTP complex (Fig. 2, I and J) (26-28). Both PICs adopt the previously observed open conformation (28). The stable association of Nsp1 in the cell with multiple different intermediates states of translation initiation besides empty $40 \mathrm{~S}$ ribosomal complexes is in agreement with the proposed role of Nsp1 as an inhibitor of translation initiation (15).

The Nsp1-bound 80S complexes could be divided into two major populations of translationally inactive ribosomes. The first population (Fig. 2, K and L, and fig. S4, A to E) contained the protein coiled-coil domain containing short open reading frame 124 (CCDC124), a homolog of the ribosome protection and translation recovery factor Lso2 in Saccharomyces cerevisiae (29). A similar complex of inactive $80 \mathrm{~S}$ ribosomes bound to $\mathrm{CCDC124}$ was recently described (30). In addition to the known hibernation complex, a subpopulation of the 
CCDC124-bound 80S contained also the ribosome recycling factor and ABC-type ATPase ABCE1 (31-33) and the class I translation termination factor eRF1 in an unusual conformation (fig. S4, C to E). The previously unresolved, flexible Cterminal part of CCDC124 was stably bound to the ribosomal A-site in this complex. This sub-population might represent a previously unidentified ribosome recycling-like state.

The second major population of Nsp1-bound $80 \mathrm{~S}$ ribosomes (Fig. 2, M and N) lacked CCDC124, but contained the cell growth regulating nucleolar protein LYAR, which has been implicated in processing of pre-rRNA and in negative regulation of antiviral innate immune responses $(34,35)$. We found the $\mathrm{C}$ terminus of LYAR occupying the ribosomal Asite, similar to CCDC124 (Fig. 2M and fig. S4, F and G). Furthermore, we identified a subpopulation among the LYARbound inactive $80 \mathrm{~S}$ ribosomes that contained a ternary eEF1A-GTP-tRNA complex (Fig. $2 \mathrm{~N}$ and fig. S4, H to K). This ternary complex was in an unusual conformation, with the anticodon loop contacting an $\alpha$-helix of the LYAR $\mathrm{C}$ terminus. Such a complex has not been previously described and its functional relevance is unknown.

Taken together, we found Nsp1 bound to the mRNA entry channel of a unique set of translationally inactive $80 \mathrm{~S}$ ribosomes, among which were unusual complexes. It is unclear, whether these are a result of the presence of Nsp1, or whether they occur naturally and have an increased affinity for Nsp1 due to their distinct conformation or lack of mRNA.

All observed ribosomal complexes displayed the same binding mode of Nsp1 to the 40S subunit, in which the Cterminal domain of Nsp1 (Nsp1-C) is rigidly bound inside the mRNA entry channel. Here, it interacts with the rRNA helix h18, with the ribosomal protein uS5 of the 40S body and with uS3 of the $40 \mathrm{~S}$ head. The local resolution of $2.6 \AA$ (fig. S3) allowed for a detailed analysis of the molecular interactions of Nsp1 with the ribosome (Fig. 3A).

The shorter, first $\alpha$-helix of Nsp1-C ( $\alpha 1$; residues 154-160) interacts with uS3 and uS5. The helix is followed by a short loop, which contains the essential $\mathrm{KH}$ motif that interacts with h18. Notably, this part of h18 belongs to the so-called "530-loop", which actively participates in ribosomal decoding and has been reported to resemble a conserved structural motif in the 3 '-UTR of beta-CoVs (36). The second, larger $\alpha$-helix of Nsp1-C ( $\alpha 2$; residues 166-179) also interacts with rRNA h18 and connects back to uS5 at its C-terminal end. The two helices stabilize each other through hydrophobic interactions. The electrostatic potential on the Nsp1-C surface displays three major patches (Fig. 3B). A negatively charged patch on $\alpha 1$ facing positively charged residues on uS3, a positively charged patch on $\alpha 2$ facing the phosphate backbone of h18, and a hydrophobic patch at the $\alpha 1-\alpha 2$ interface, which is exposed to hydrophobic residues on uS5. In addition to the matching surface charge, the shape of Nsp1-C matches the shape of the mRNA channel and completely overlaps the regular mRNA path (Fig. 3, C and D). Together, this explains the strong inhibitory effect on translation observed in vitro and in vivo. A key interaction is established through the $\mathrm{KH}$ motif, which binds to a distinct site on rRNA helix h18 (Fig. 3, C and E); K164 of Nsp1 inserts into a negatively charged pocket, constituted mainly by the phosphate backbone of rRNA bases G625 and U630, whereas H165 stacks in between U607 and U630. The base U630 is stabilized in this position through interaction with the backbone of G168 of Nsp1. Further interactions involve R171 and R175 of Nsp1, which form salt bridges to the backbone phosphates of G601, C607, A605 and G606 of h18 (Fig. 3F). The interactions of Nsp1-C and uS3 are established through salt bridges and hydrogen bonds between D152, E155, E159 of Nsp1 and R116, R143 and M150 of uS3 (Fig. 3G). The interactions of Nsp1-C with uS5 occur within a hydrophobic surface of $\sim 440 \AA^{2}$ involving residues Y154, F157, W161, T170, L173, M174, L177 of Nsp1 and residues V106, I109, P111, T122, F124, V147, I151 of uS5 (Fig. 3H). Taken together, specific molecular contacts (summarized in Fig. 3I) rigidly anchor Nsp1 and thereby obstruct the mRNA entry channel.

Type-I interferon induction and signaling represents one of the major innate anti-viral defense pathways, ultimately leading to the induction of several hundred anti-viral interferon-stimulated genes (ISGs) (37). Coronavirus infections are sensed by RIG-I which activates this defense system (37, 38). To assess the effects of SARS-CoV-2 Nsp1 on the interferon system, we stimulated HEK293T cells with Sendai Virus (SeV), a well-known trigger of RIG-I-dependent signaling (39, 40). Expression of Nsp1 completely abrogated the translation of Firefly Luciferase controlled by human interferon-beta (IFN- $\beta$ ) promoter, whereas the Nsp1-mt had no significant effect (Fig. 4A and fig. S5A), confirming the results of the in vitro translation assays. Rabies virus P protein (41) and SARSCoV-2 Nsp7 were used as positive and negative control, respectively. After stimulation with $\mathrm{SeV}$, the protein levels of endogenous IFN- $\beta$, IFN- $\lambda 1$ and interleukin-8 (IL-8) (Fig. 4B and fig. S5, B and C) in the supernatant of Nsp1 expressing cells were drastically reduced, although transcription of the corresponding mRNAs was induced. Again, Nsp1-mt showed no inhibitory effect. Expression of Luciferase driven by the interferon stimulated response element (ISRE), which is part of the promoter of most ISGs, was effectively shut down by Nsp1 but not by Nsp1-mt in a dose-dependent manner (Fig. 4, $\mathrm{C}$ and $\mathrm{D}$, and fig. S5D). SARS CoV-2 Nsp7 and Measles virus $\mathrm{V}$ protein $(\mathrm{MeV} \mathrm{V})(40,42)$ served as negative and positive controls, respectively. In line with this, Nsp1 but not Nsp1-mt suppressed the induction of endogenous RIG-I and ISG15 upon IFN- $\beta$ stimulation on the protein but not the mRNA level (Fig. 4E).

Notably, not all innate immune responses require active 
translation for function. For example autophagy is barely affected by the expression of Nsp1 or its mutant (fig. S5E) even upon induction with Rapamycin (43). Tripartite Motif Protein 32 (TRIM32) was used as a positive control (44). Taken together, these results demonstrate that SARS-CoV-2 Nsp1 almost completely prevents translation not only of interferons and other pro-inflammatory cytokines but also of interferonstimulated anti-viral ISGs.

Our data establish that one of the major immune evasion factors of SARS-CoV-2, Nsp1, efficiently interferes with the cellular translation machinery resulting in a shut-down of host protein production. Thus, major parts of the innate immune system, that depend on translation of antiviral defense factors such as IFN- $\beta$ or RIG-I (45) are disarmed. Although SARS-CoV-2 encodes additional potential inhibitors of the innate immune defenses, a loss of Nsp1 function may render the virus vulnerable toward immune clearance. Thus, our data may provide a starting point for rational structure-based drug design, targeting the Nsp1-ribosome interaction.

However, important questions remain to be addressed. For example, how can the virus overcome the Nsp1-mediated block of translation for the production of its own viral proteins? Common structural features present in the $5^{\prime}$ untranslated region of all SARS-CoV mRNAs, may help to circumvent the ribosome blockage by Nsp1 (46).

\section{REFERENCES AND NOTES}

1. S. R. Weiss, S. Navas-Martin, Coronavirus pathogenesis and the emerging pathogen severe acute respiratory syndrome coronavirus. Microbiol. Mol. Biol. Rev. 69, 635-664 (2005). doi:10.1128/MMBR.69.4.635-664.2005 Medline

2. P. C. Woo, S. K. P. Lau, C. S. F. Lam, C. C. Y. Lau, A. K. L. Tsang, J. H. N. Lau, R. Bai, J. L. L. Teng, C. C. C. Tsang, M. Wang, B.-J. Zheng, K.-H. Chan, K.-Y. Yuen, Discovery of seven novel mammalian and avian coronaviruses in the genus Deltacoronavirus supports bat coronaviruses as the gene source of Alphacoronavirus and Betacoronavirus and avian coronaviruses as the gene source of Gammacoronavirus and Deltacoronavirus. J. Virol. 86, 3995-4008 (2012). doi:10.1128/JVI.06540-11 Medline

3. J. Cui, F. Li, Z. L. Shi, Origin and evolution of pathogenic coronaviruses. Nat. Rev. Microbiol. 17, 181-192 (2019). doi:10.1038/s41579-018-0118-9 Medline

4. L. van der Hoek, Human coronaviruses: What do they cause? Antivir. Ther. 12, 651658 (2007). Medline

5. L. van der Hoek, K. Pyrc, B. Berkhout, Human coronavirus NL63, a new respiratory virus. FEMS Microbiol. Rev. 30, 760-773 (2006). doi:10.1111/i.15746976.2006.00032.x Medline

6. E. de Wit, N. van Doremalen, D. Falzarano, V. J. Munster, SARS and MERS: Recent insights into emerging coronaviruses. Nat. Rev. Microbiol. 14, 523-534 (2016). doi:10.1038/nrmicro.2016.81 Medline

7. D. Wang, B. Hu, C. Hu, F. Zhu, X. Liu, J. Zhang, B. Wang, H. Xiang, Z. Cheng, Y. Xiong, Y. Zhao, Y. Li, X. Wang, Z. Peng, Clinical characteristics of 138 hospitalized patients with 2019 novel coronavirus-infected pneumonia in Wuhan, China. JAMA 323, 1061-1069 (2020). doi:10.1001/jama.2020.1585 Medline

8. Y. X. Lim, Y. L. Ng, J. P. Tam, D. X. Liu, Human coronaviruses: A review of virus-host interactions. Diseases 4, 26 (2016). doi:10.3390/diseases4030026 Medline

9. P. Zhou, X.-L. Yang, X.-G. Wang, B. Hu, L. Zhang, W. Zhang, H.-R. Si, Y. Zhu, B. Li, C.L. Huang, H.-D. Chen, J. Chen, Y. Luo, H. Guo, R.-D. Jiang, M.-Q. Liu, Y. Chen, X.-R. Shen, X. Wang, X.-S. Zheng, K. Zhao, Q.-J. Chen, F. Deng, L.-L. Liu, B. Yan, F.-X. Zhan, Y.-Y. Wang, G.-F. Xiao, Z.-L. Shi, A pneumonia outbreak associated with a new coronavirus of probable bat origin. Nature 579, 270-273 (2020). doi:10.1038/s41586-020-2012-7 Medline
10. P. S. Masters, The molecular biology of coronaviruses. Adv. Virus Res. 66, 193292 (2006). doi:10.1016/S0065-3527(06)66005-3 Medline

11. L. Lei, S. Ying, L. Baojun, Y. Yi, H. Xiang, S. Wenli, S. Zounan, G. Deyin, Z. Qingyu, L. Jingmei, C. Guohui, Attenuation of mouse hepatitis virus by deletion of the LLRKXGXKG region of Nsp1. PLOS ONE 8, e61166 (2013). doi:10.1371/journal.pone.0061166 Medline

12. Y. Tohya, K. Narayanan, W. Kamitani, C. Huang, K. Lokugamage, S. Makino, Suppression of host gene expression by nspl proteins of group 2 bat coronaviruses. J. Virol. 83, 5282-5288 (2009). doi:10.1128/JVI.02485-08 Medline

13. K. Narayanan, C. Huang, K. Lokugamage, W. Kamitani, T. Ikegami, C.-T. K. Tseng, S. Makino, Severe acute respiratory syndrome coronavirus nspl suppresses host gene expression, including that of type I interferon, in infected cells. J. Virol. 82. 4471-4479 (2008). doi:10.1128/JVI.02472-07 Medline

14. C. Huang, K. G. Lokugamage, J. M. Rozovics, K. Narayanan, B. L. Semler, S. Makino, Alphacoronavirus transmissible gastroenteritis virus nspl protein suppresses protein translation in mammalian cells and in cell-free HeLa cell extracts but not in rabbit reticulocyte lysate. J. Virol. 85, 638-643 (2011). doi:10.1128/JVl.0180610 Medline

15. K. G. Lokugamage, K. Narayanan, C. Huang, S. Makino, Severe acute respiratory syndrome coronavirus protein nspl is a novel eukaryotic translation inhibitor that represses multiple steps of translation initiation. J. Virol. 86, 13598-13608 (2012). doi:10.1128/JVI.01958-12 Medline

16. W. Kamitani, C. Huang, K. Narayanan, K. G. Lokugamage, S. Makino, A two-pronged strategy to suppress host protein synthesis by SARS coronavirus Nspl protein. Nat. Struct. Mol. Biol. 16, 1134-1140 (2009). doi:10.1038/nsmb.1680 Medline

17. C. Huang, K. G. Lokugamage, J. M. Rozovics, K. Narayanan, B. L. Semler, S. Makino, SARS coronavirus nspl protein induces template-dependent endonucleolytic cleavage of mRNAs: Viral mRNAs are resistant to nsp1-induced RNA cleavage. PLOS Pathog. 7, e1002433 (2011). doi:10.1371/journal.ppat.1002433 Medline

18. M. G. Wathelet, M. Orr, M. B. Frieman, R. S. Baric, Severe acute respiratory syndrome coronavirus evades antiviral signaling: Role of nspl and rational design of an attenuated strain. J. Virol. 81, 11620-11633 (2007). doi:10.1128/JVI.0070207 Medline

19. C. Wu, Y. Liu, Y. Yang, P. Zhang, W. Zhong, Y. Wang, Q. Wang, Y. Xu, M. Li, X. Li, M. Zheng, L. Chen, H. Li, Analysis of therapeutic targets for SARS-CoV-2 and discovery of potential drugs by computational methods. Acta Pharm. Sin. B 10 , 766-788 (2020). doi:10.1016/j.apsb.2020.02.008 Medline

20. A. R. Jauregui, D. Savalia, V. K. Lowry, C. M. Farrell, M. G. Wathelet, Identification of residues of SARS-CoV nspl that differentially affect inhibition of gene expression and antiviral signaling. PLOS ONE 8, e62416 (2013). doi:10.1371/journal.pone.0062416 Medline

21. M. S. Almeida, M. A. Johnson, T. Herrmann, M. Geralt, K. Wüthrich, Novel $\beta$-barrel fold in the nuclear magnetic resonance structure of the replicase nonstructural protein 1 from the severe acute respiratory syndrome coronavirus. J. Virol. 81 , 3151-3161 (2007). doi:10.1128/JVI.01939-06 Medline

22. F. Schluenzen, A. Tocilj, R. Zarivach, J. Harms, M. Gluehmann, D. Janell, A. Bashan, H. Bartels, I. Agmon, F. Franceschi, A. Yonath, Structure of functionally activated small ribosomal subunit at $3.3 \AA$ resolution. Cell 102, 615-623 (2000). doi:10.1016/S0092-8674(00)00084-2 Medline

23. L. A. Passmore, T. M. Schmeing, D. Maag, D. J. Applefield, M. G. Acker, M. A. Algire J. R. Lorsch, V. Ramakrishnan, The eukaryotic translation initiation factors elF1 and elF1A induce an open conformation of the 40 S ribosome. Mol. Cell 26, 41-50 (2007). doi:10.1016/j.molcel.2007.03.018 Medline

24. M. Ameismeier, J. Cheng, O. Berninghausen, R. Beckmann, Visualizing late states of human 40S ribosomal subunit maturation. Nature 558, 249-253 (2018). doi:10.1038/s41586-018-0193-0 Medline

25. A. Heuer, E. Thomson, C. Schmidt, O. Berninghausen, T. Becker, E. Hurt, R. Beckmann, Cryo-EM structure of a late pre-40S ribosomal subunit from Saccharomyces cerevisiae. elife 6, e30189 (2017). doi:10.7554/eLife.30189 Medline

26. A. des Georges, V. Dhote, L. Kuhn, C. U. T. Hellen, T. V. Pestova, J. Frank, Y. Hashem, Structure of mammalian elF3 in the context of the 43S preinitiation complex. Nature 525, 491-495 (2015). doi:10.1038/nature14891 Medline

27. Y. Hashem, A. des Georges, V. Dhote, R. Langlois, H. Y. Liao, R. A. Grassucci, C. U. 
T. Hellen, T. V. Pestova, J. Frank, Structure of the mammalian ribosomal 43S preinitiation complex bound to the scanning factor DHX29. Cell 153, 1108-1119 (2013). doi:10.1016/i.cell.2013.04.036 Medline

28. J. L. Llácer, T. Hussain, L. Marler, C. E. Aitken, A. Thakur, J. R. Lorsch, A. G. Hinnebusch, V. Ramakrishnan, Conformational differences between open and closed states of the eukaryotic translation initiation complex. Mol. Cell 59, 399412 (2015). doi:10.1016/i.molcel.2015.06.033 Medline

29. Y. J. Wang, P. P. Vaidyanathan, M. F. Rojas-Duran, N. D. Udeshi, K. M. Bartoli, S. A. Carr, W. V. Gilbert, Lso2 is a conserved ribosome-bound protein required for translational recovery in yeast. PLOS Biol. 16, e2005903 (2018). doi:10.1371/journal.pbio.2005903 Medline

30. J. N. Wells, R. Buschauer, T. Mackens-Kiani, K. Best, H. Kratzat, O. Berninghausen, T. Becker, W. Gilbert, J. Cheng, R. Beckmann, Structure and function of yeast Lso2 and human $\mathrm{CCDC124}$ bound to hibernating ribosomes. bioRxiv 944066 [Preprint]. 12 February 2020; https://doi.org/10.1101/2020.02.12.944066.

31. T. Becker, S. Franckenberg, S. Wickles, C. J. Shoemaker, A. M. Anger, J.-P. Armache, H. Sieber, C. Ungewickell, O. Berninghausen, I. Daberkow, A. Karcher, M. Thomm, K.-P. Hopfner, R. Green, R. Beckmann, Structural basis of highly conserved ribosome recycling in eukaryotes and archaea. Nature 482, 501-506 (2012). doi:10.1038/nature10829 Medline

32. A. Preis, A. Heuer, C. Barrio-Garcia, A. Hauser, D. E. Eyler, O. Berninghausen, R. Green, T. Becker, R. Beckmann, Cryoelectron microscopic structures of eukaryotic translation termination complexes containing eRF1-eRF3 or eRF1ABCE1. Cell Rep. 8, 59-65 (2014). doi:10.1016/i.celrep.2014.04.058 Medline

33. A. Brown, S. Shao, J. Murray, R. S. Hegde, V. Ramakrishnan, Structural basis for stop codon recognition in eukaryotes. Nature 524, 493-496 (2015). doi:10.1038/nature14896 Medline

34. N. Miyazawa, H. Yoshikawa, S. Magae, H. Ishikawa, K. Izumikawa, G. Terukina, A. Suzuki, S. Nakamura-Fujiyama, Y. Miura, T. Hayano, W. Komatsu, T. Isobe, N. Takahashi, Human cell growth regulator Ly-1 antibody reactive homologue accelerates processing of preribosomal RNA. Genes Cells 19, 273-286 (2014). doi:10.1111/gtc.12129 Medline

35. C. Yang, X. Liu, T. Cheng, R. Xiao, Q. Gao, F. Ming, M. Jin, H. Chen, H. Zhou, LYAR suppresses beta interferon induction by targeting phosphorylated interferon regulatory factor 3. J. Virol. 93, e00769-19 (2019). doi:10.1128/JVl.00769-19 Medline

36. M. P. Robertson, H. Igel, R. Baertsch, D. Haussler, M. Ares Jr., W. G. Scott, The structure of a rigorously conserved RNA element within the SARS virus genome. PLOS Biol. 3, e5 (2005). doi:10.1371/journal.pbio.0030005 Medline

37. K. M. Sparrer, M. U. Gack, Intracellular detection of viral nucleic acids. Curr. Opin. Microbiol. 26, 1-9 (2015). doi:10.1016/j.mib.2015.03.001 Medline

38. Y. Hu, W. Li, T. Gao, Y. Cui, Y. Jin, P. Li, Q. Ma, X. Liu, C. Cao, The severe acute respiratory syndrome coronavirus nucleocapsid inhibits type I interferon production by interfering with TRIM25-mediated RIG-I ubiquitination. J. Virol. 91, e02143-16 (2017). doi:10.1128/JVI.02143-16 Medline

39. L. Strähle, J.-B. Marq, A. Brini, S. Hausmann, D. Kolakofsky, D. Garcin, Activation of the beta interferon promoter by unnatural Sendai virus infection requires RIG-I and is inhibited by viral C proteins. J. Virol. 81, 12227-12237 (2007). doi:10.1128/JVI.01300-07 Medline

40. K. M. Sparrer, C. K. Pfaller, K. K. Conzelmann, Measles virus $C$ protein interferes with Beta interferon transcription in the nucleus. J. Virol. 86, 796-805 (2012). doi:10.1128/JVI.05899-11 Medline

41. K. Brzózka, S. Finke, K. K. Conzelmann, Identification of the rabies virus alpha/beta interferon antagonist: Phosphoprotein $\mathrm{P}$ interferes with phosphorylation of interferon regulatory factor 3. J. Virol. 79, 7673-7681 (2005). doi:10.1128/JVI.79.12.7673-7681.2005 Medline

42. P. Devaux, V. von Messling, W. Songsungthong, C. Springfeld, R. Cattaneo, Tyrosine 110 in the measles virus phosphoprotein is required to block STAT1 phosphorylation. Virology 360, 72-83 (2007). doi:10.1016/j.virol.2006.09.049 Medline

43. K. M. J. Sparrer, S. Gableske, M. A. Zurenski, Z. M. Parker, F. Full, G. J. Baumgart, J. Kato, G. Pacheco-Rodriguez, C. Liang, O. Pornillos, J. Moss, M. Vaughan, M. U. Gack, TRIM23 mediates virus-induced autophagy via activation of TBK1. Nat. Microbiol. 2, 1543-1557 (2017). doi:10.1038/s41564-017-0017-2 Medline

44. M. Di Rienzo, M. Piacentini, G. M. Fimia, A TRIM32-AMBRA1-ULK1 complex initiates the autophagy response in atrophic muscle cells. Autophagy 15, 1674-1676 (2019). doi:10.1080/15548627.2019.1635385 Medline

45. M. Z. Tay, C. M. Poh, L. Rénia, P. A. MacAry, L. F. P. Ng, The trinity of COVID-19: Immunity, inflammation and intervention. Nat. Rev. Immunol. 20, 363-374 (2020). doi:10.1038/s41577-020-0311-8 Medline

46. T. Tanaka, W. Kamitani, M. L. DeDiego, L. Enjuanes, Y. Matsuura, Severe acute respiratory syndrome coronavirus nspl facilitates efficient propagation in cells through a specific translational shutoff of host mRNA. J. Virol. 86, 11128-11137 (2012). doi:10.1128/JVI.01700-12 Medline

47. V. Bhaskar, A. Graff-Meyer, A. D. Schenk, S. Cavadini, O. von Loeffelholz, S. K. Natchiar, C. G. Artus-Revel, H.-R. Hotz, G. Bretones, B. P. Klaholz, J. A. Chao, Dynamics of uS19 C-terminal tail during the translation elongation cycle in human ribosomes. Cell Rep. 31, 107473 (2020). doi:10.1016/i.celrep.2020.03.037 Medline

48. S. Matheisl, O. Berninghausen, T. Becker, R. Beckmann, Structure of a human translation termination complex. Nucleic Acids Res. 43, 8615-8626 (2015). doi:10.1093/nar/gkv909 Medline

49. C. Sidrauski, A. M. McGeachy, N. T. Ingolia, P. Walter, The small molecule ISRIB reverses the effects of elF $2 \alpha$ phosphorylation on translation and stress granule assembly. eLife 4, e05033 (2015). doi:10.7554/elife.05033 Medline

50. A. Sharma, M. Mariappan, S. Appathurai, R. S. Hegde, In vitro dissection of protein translocation into the mammalian endoplasmic reticulum. Methods Mol. Biol. 619, 339-363 (2010). doi:10.1007/978-1-60327-412-8_20 Medline

51. S. Q. Zheng, E. Palovcak, J.-P. Armache, K. A. Verba, Y. Cheng, D. A. Agard, MotionCor2: Anisotropic correction of beam-induced motion for improved cryoelectron microscopy. Nat. Methods 14, 331-332 (2017). doi:10.1038/nmeth.4193 Medline

52. K. Zhang, Gctf: Real-time CTF determination and correction. J. Struct. Biol. 193, 112 (2016). doi:10.1016/i.jsb.2015.11.003 Medline

53. A. Rohou, N. Grigorieff, CTFFIND4: Fast and accurate defocus estimation from electron micrographs. J. Struct. Biol. 192, 216-221 (2015). doi:10.1016/i.jsb.2015.08.008 Medline

54. J. Zivanov, T. Nakane, B. O. Forsberg, D. Kimanius, W. J. H. Hagen, E. Lindahl, S. H. W. Scheres, New tools for automated high-resolution cryo-EM structure determination in RELION-3. elife 7, e42166-e42166 (2018). doi:10.7554/eLife.42166 Medline

55. J. Zivanov, T. Nakane, S. H. W. Scheres, Estimation of high-order aberrations and anisotropic magnification from cryo-EM data sets in RELION-3.1. IUCrJ 7, 253267 (2020). doi:10.1107/S2052252520000081 Medline

56. A. Punjani, J. L. Rubinstein, D. J. Fleet, M. A. Brubaker, cryoSPARC: Algorithms for rapid unsupervised cryo-EM structure determination. Nat. Methods 14, 290-296 (2017). doi:10.1038/nmeth.4169 Medline

57. T. Burnley, C. M. Palmer, M. Winn, Recent developments in the CCP-EM software suite. Acta Crystallogr. D Struct. Biol. 73, 469-477 (2017). doi:10.1107/S2059798317007859 Medline

58. P. Emsley, K. Cowtan, Coot: Model-building tools for molecular graphics. Acta Crystallogr. D Biol. Crystallogr. 60, 2126-2132 (2004) doi:10.1107/S0907444904019158 Medline

59. P. Emsley, B. Lohkamp, W. G. Scott, K. Cowtan, Features and development of Coot. Acta Crystallogr. D Biol. Crystallogr. 66, 486-501 (2010). doi:10.1107/S0907444910007493 Medline

60. S. Sarkar, S. Witham, J. Zhang, M. Zhenirovskyy, W. Rocchia, E. Alexov, DelPhi Web Server: A comprehensive online suite for electrostatic calculations of biological macromolecules and their complexes. Commun. Comput. Phys. 13, 269-284 (2013). doi:10.4208/cicp.300611.201011s Medline

61. H. Kratzat, T. Mackens-Kiani, M. Ameismeier, J. Cheng, E. Dacheux, A. Namane, 0. Berninghausen, M. Fromont-Racine, T. Becker, R. Beckmann, Structural inventory of native ribosomal ABCE1-43S pre-initiation complexes. bioRxiv 194902 [Preprint]. 9 July 2020; https://doi.org/10.1101/2020.07.09.194902.

62. T. D. Goddard, C. C. Huang, E. C. Meng, E. F. Pettersen, G. S. Couch, J. H. Morris, T. E. Ferrin, UCSF ChimeraX: Meeting modern challenges in visualization and analysis. Protein Sci. 27, 14-25 (2018). doi:10.1002/pro.3235 Medline 


\section{ACKNOWLEDGMENTS}

Sendai virus was kindly provided by Georg Kochs and Daniel Sauter. Luciferase Reporter constructs and RV P antibody were provided by Karl-Klaus Conzelmann. We thank Susanne Engelhart, Kerstin Regensburger, Martha Meyer, Regina Burger, Nicole Schrott, Daniela Krnavek, Miwako Kösters,

Charlotte Ungewickell and Susanne Rieder for excellent technical assistance. Funding: This study was supported by a Ph.D. fellowship by Boehringer Ingelheim Fonds to R.Bu., grants by the DFG to R.Be (SFB/TRR-174, BE1814/151, BE1814/1-1), grants by the DFG to K.S. (CRC-1279, SPP-1923, SP1600/4-1), grants by the DFG and BMBF to FK (CRC-1279, SPP-1923, RestrictSARS-CoV2) as well as intramural funding by University Ulm Medical Center (L.SBN.0150) to K.S. Author contributions: R.Be., K.S., M.T., R.Bu. and M.A. designed the study; O.B. collected cryo-EM data, M.T., R.Bu. and M.A. prepared cryo-EM samples and processed cryo-EM data; R.Bu., M.A. and J.C. built molecular models; T.D. performed in vitro translation assays with help from H.K.; M.T. generated plasmids and performed protein purifications and binding assays; T.MK. and H.K. performed co-sedimentation assays; T.F. performed mass spectrometry analysis; L.K., M.Hi., M.Ha and J.H.S. performed immune inhibition assays. C.S. contributed and designed codon-optimized plasmids. M.T., R.Bu., M.A., T.B., F.K., K.S. and R.Be. wrote the manuscript, with comments from all authors. Competing interests: Authors declare no competing interests. Data and materials availability: Cryo-EM volumes and molecular models have been deposited at the Electron Microscopy Data Bank and Protein Data Bank with accession codes EMD-11276, EMD-11288, EMD-11289, EMD-11292, EMD-11299, EMD-11301, EMD-11310, EMD-11325, EMD-11335 and PDB-6ZLW, PDB-6ZM7, PDB-6ZME, PDB-6ZMI, PDB-6ZM0, PDB-6ZMT, PDB-6ZN5, PDB-6ZON, PDB6ZP4. Materials are available from the authors on request. This work is licensed under a Creative Commons Attribution 4.0 International (CC BY 4.0) license, which permits unrestricted use, distribution, and reproduction in any medium, provided the original work is properly cited. To view a copy of this license, visit https://creativecommons.org/licenses/by/4.0/. This license does not apply to figures/photos/artwork or other content included in the article that is credited to a third party; obtain authorization from the rights holder before using such material.

\section{SUPPLEMENTARY MATERIALS}

science.sciencemag.org/cgi/content/full/science.abc8665/DC1

Materials and Methods

Figs. S1 to S5

Tables S1 and S2

References (48-62)

MDAR Reproducibility Checklist

Data S1

18 May 2020; accepted 13 July 2020

Published online 17 July 2020

10.1126/science.abc8665 
A

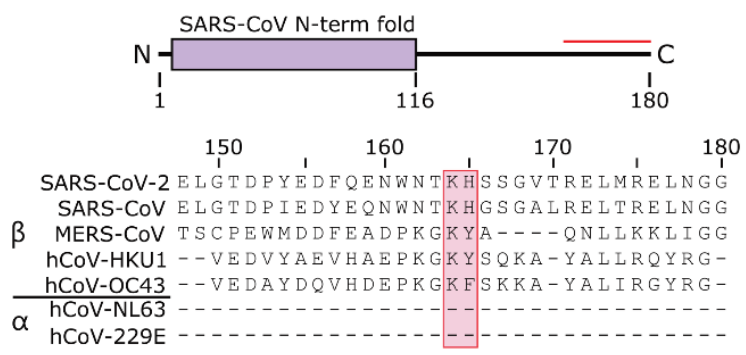

C
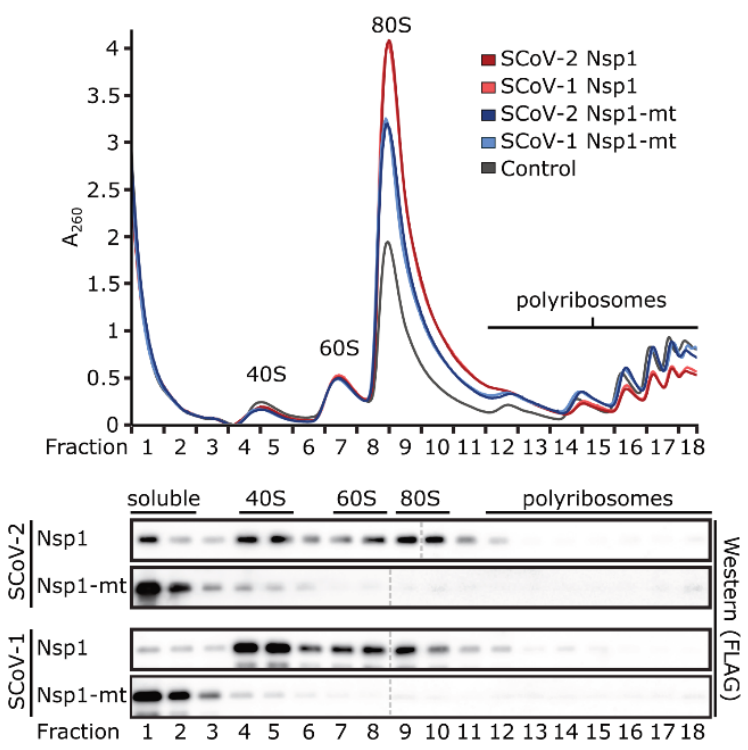

B

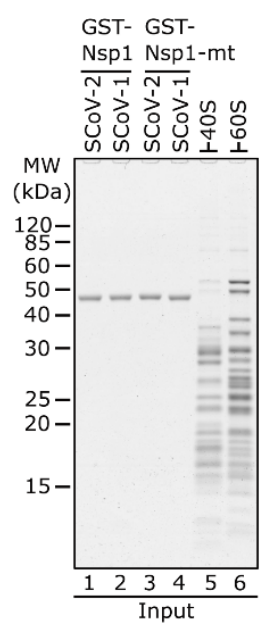

D

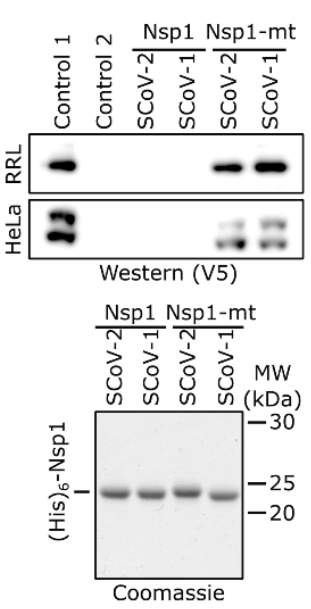

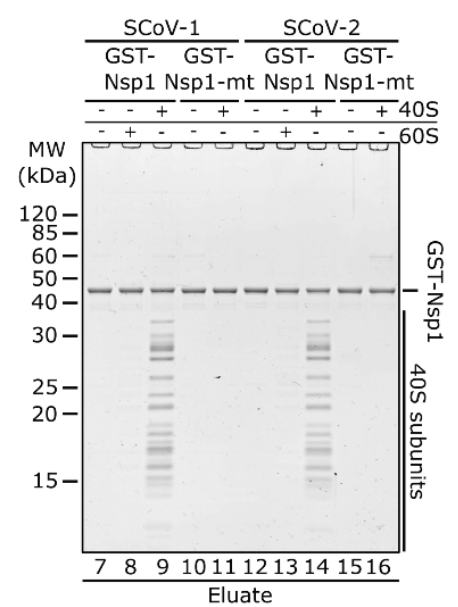

E

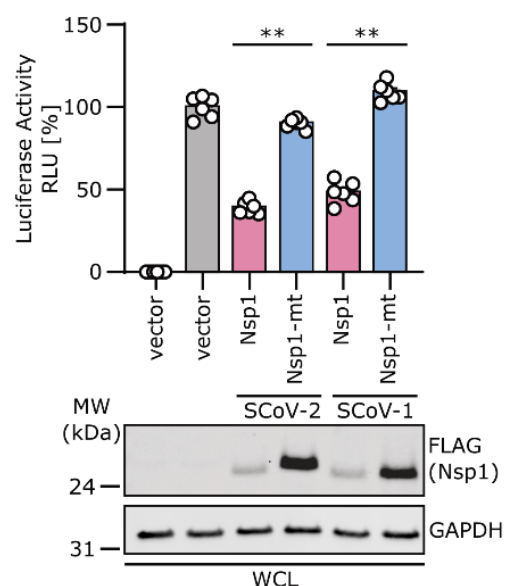

Fig. 1. Nsp1 interacts with $40 S$ ribosomal subunits and inhibits translation. (A) Domain organization of Nspl and sequence alignment of the C-terminal segment (red line) of Nspl from seven human CoVs. The KH motif is marked. (B) In vitro binding assay of GST-TEV (GST) tagged Nsp1 and Nsp1-mt from SCoV-1 and SCoV-2 with human 40S and 605 ribosomal subunits. Coomassie stained SDS-PAGE of inputs and eluates. (C) Polyribosome gradient analysis of HEK293T lysate (Control) and lysate from HEK293T cells transiently transfected with 3xFLAG tagged Nsp1 and Nsp1-mt constructs from SCoV-1 and SCoV-2 and Western blot analysis (anti-FLAG antibody, dashed lines: separate blots). (D) Western blot (top, anti-V5 antibody) and SDS-PAGE analysis (bottom) of cell-free in vitro translation of a capped reporter mRNA with rabbit reticulocytes (RRL) and HeLa S3 lysate. Controls 1 and 2, with and without capped reporter mRNA, respectively. Coomassie stained SDS-PAGE of the applied (His) $)_{6}$-TEV $\left(\mathrm{His}_{6}\right)$ tagged Nspl constructs is shown below. (E) Quantification of luciferase in HEK293T cells transfected with indicated 3xFLAG-tagged proteins and in vitro transcribed firefly luciferase mRNA. Bars represent the mean of $n=6 \pm S E M$. RLU, relative light units. Representative immunoblots of whole cell lysates (WCL) stained with anti-FLAG and anti-GAPDH). Unpaired student's $t$ test (Welch correction), ${ }^{* *}, p<0.001$. 


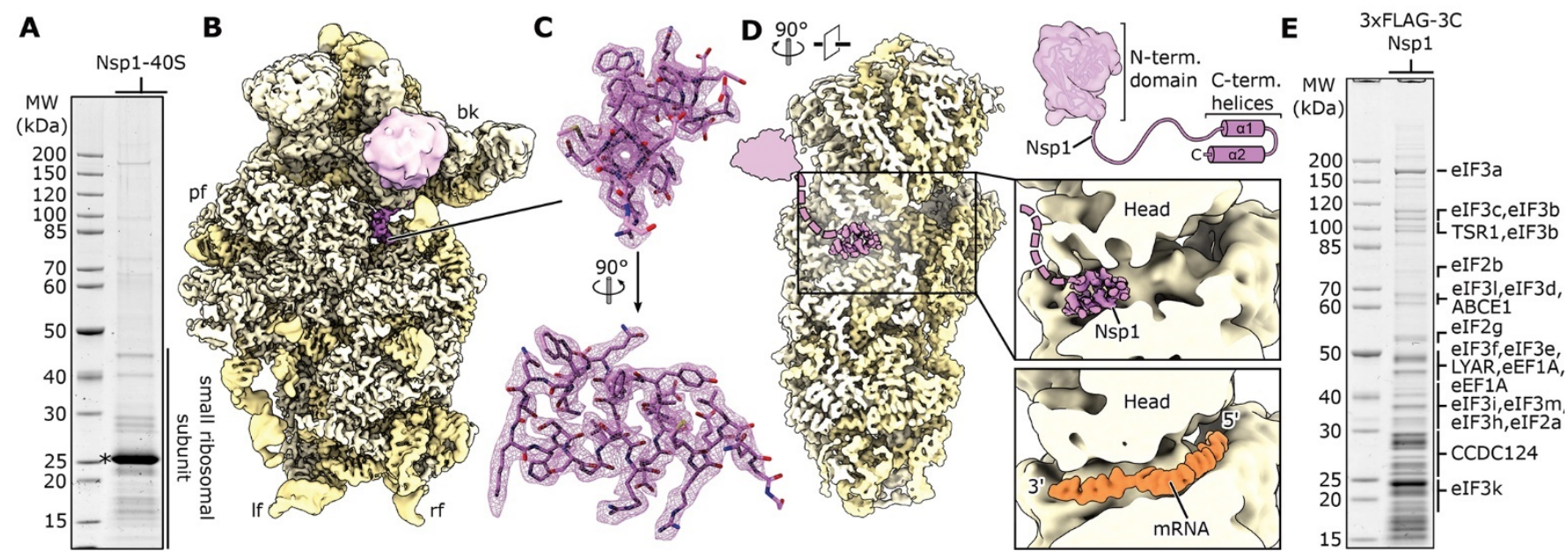

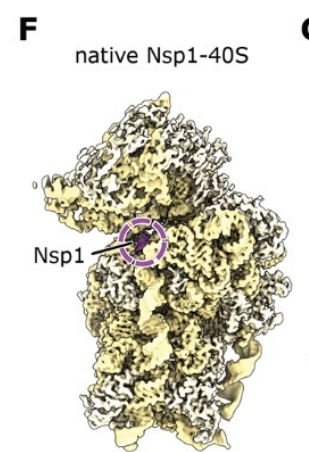

$\mathbf{K}$

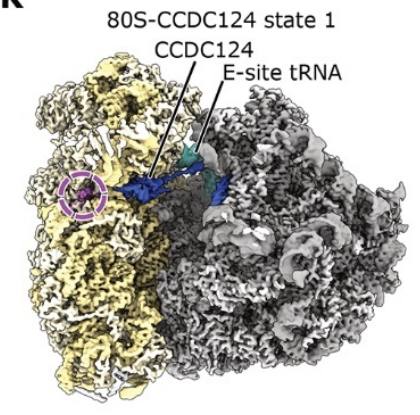

G

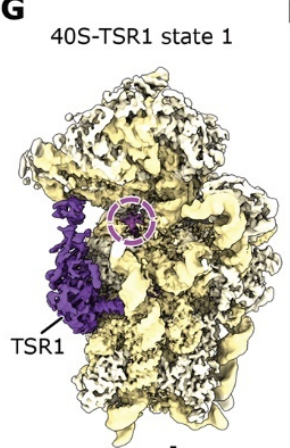

H

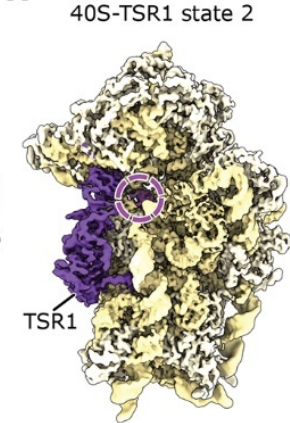

I

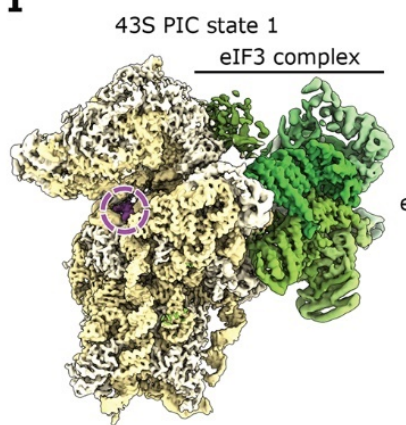

M

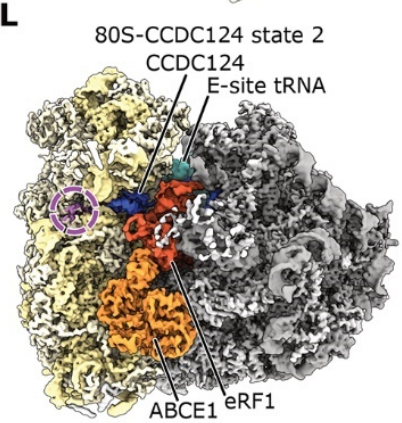

M

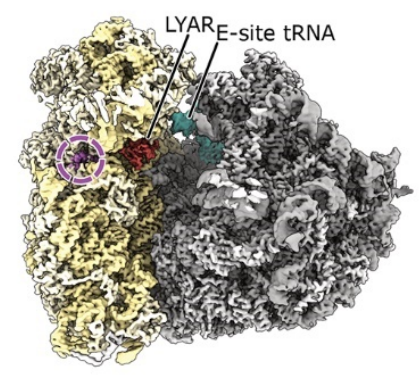

J

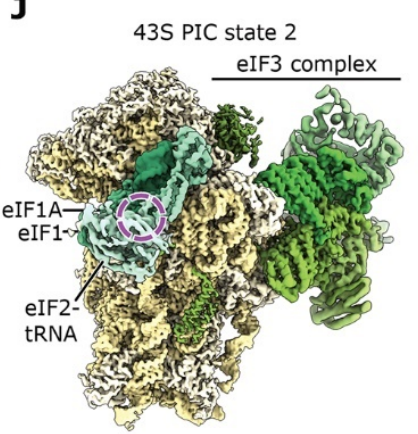

$\mathbf{N}$

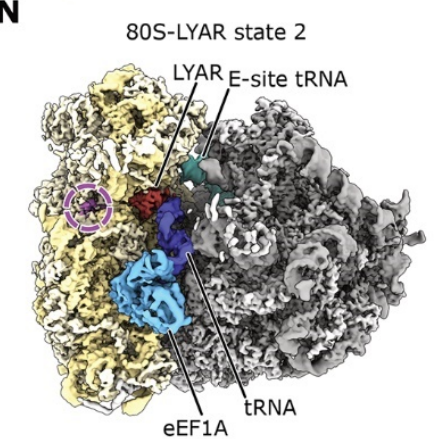

Fig. 2. Cryo-EM structures of Nsp1-bound ribosomal complexes. (A) SDS-PAGE analysis of reconstituted Nsp1-40S complexes, Nsp1 is labeled with an asterisk. (B) Reconstituted Nsp1-40S structure with Nsp1 in pink and ribosomal RNA and proteins in yellow. Additional density between uS3 and h16 assigned to the N-terminal fold of Nsp1. bk, beak; pf, platform; If, left foot; rf, right foot. (C) C-terminal helix 1 and 2 of Nsp1 with corresponding density. (D) Cross-section of the 40S highlighting the central position of Nspl within the mRNA tunnel. The putative position of the Nterminal domain of Nsp1 is schematically indicated [models based on PDB-2HSX (21) and PDB-6YOG (47)]. (E) SDS-PAGE analysis of Nsp1-ribosomal complexes affinity purified from HEK293T cells. Proteins identified in the cryo-EM structures were labeled according to mass spectrometry analysis (see data S1). (F to N) Cryo-EM maps of affinity purified Nsp1-ribosomal complexes. Additional factors are colored and labeled accordingly. 

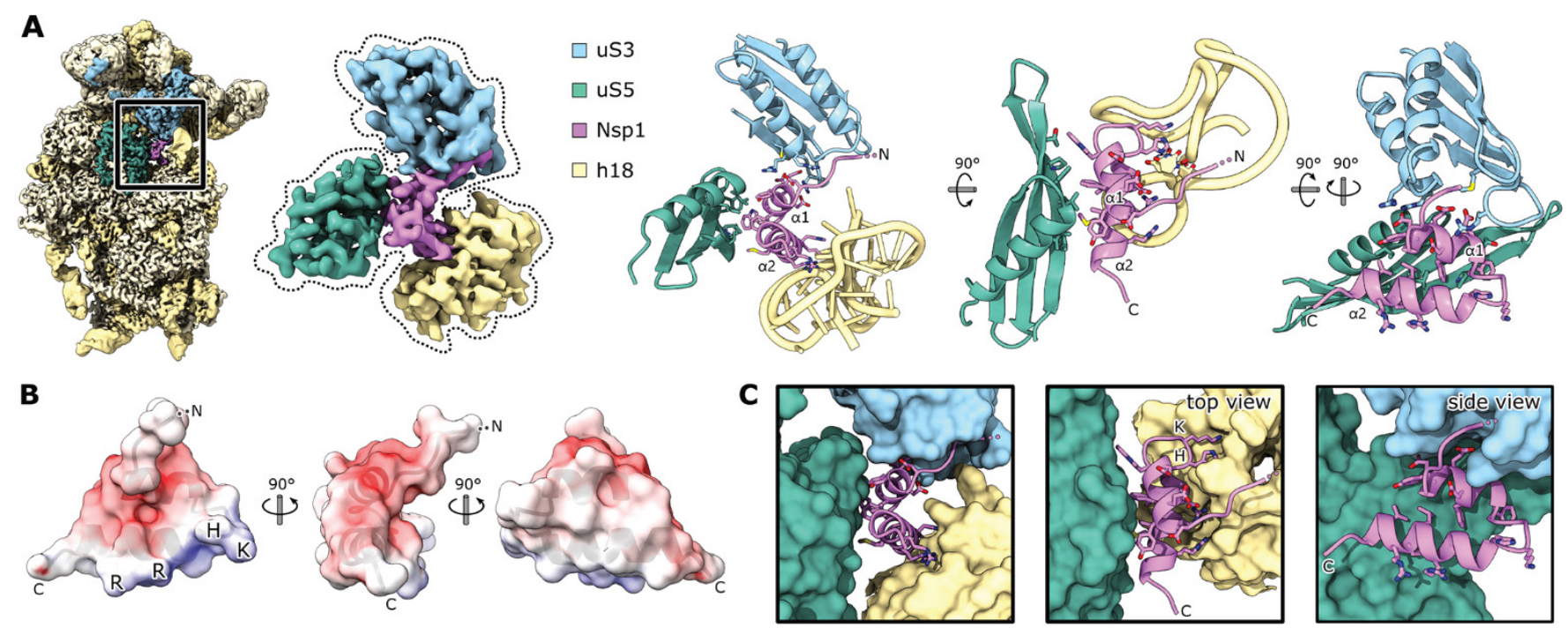

D

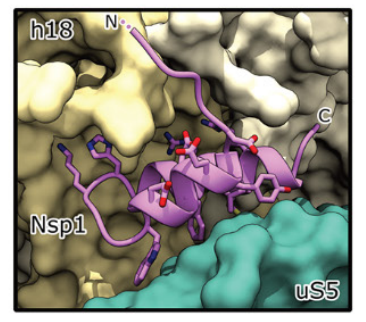

$\mathbf{F}$

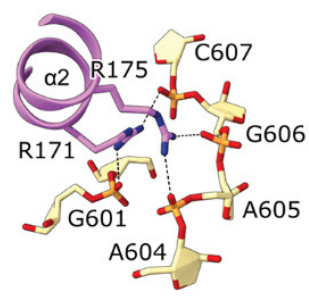

G

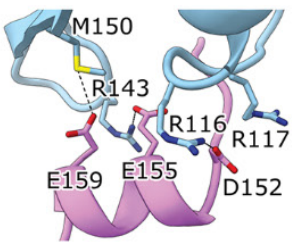

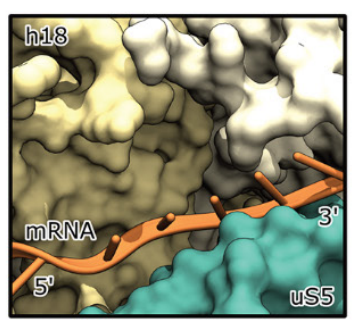

$\mathbf{H}$
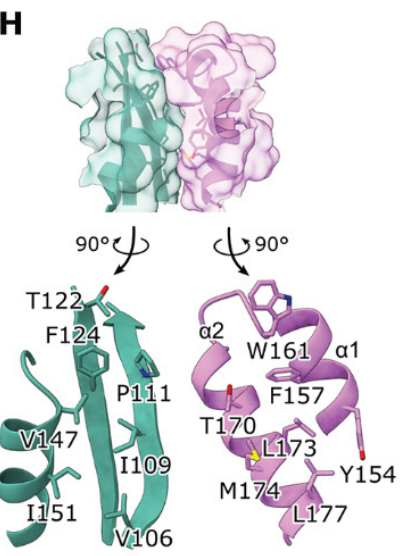

E

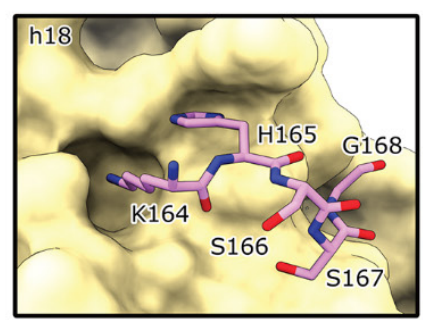

I
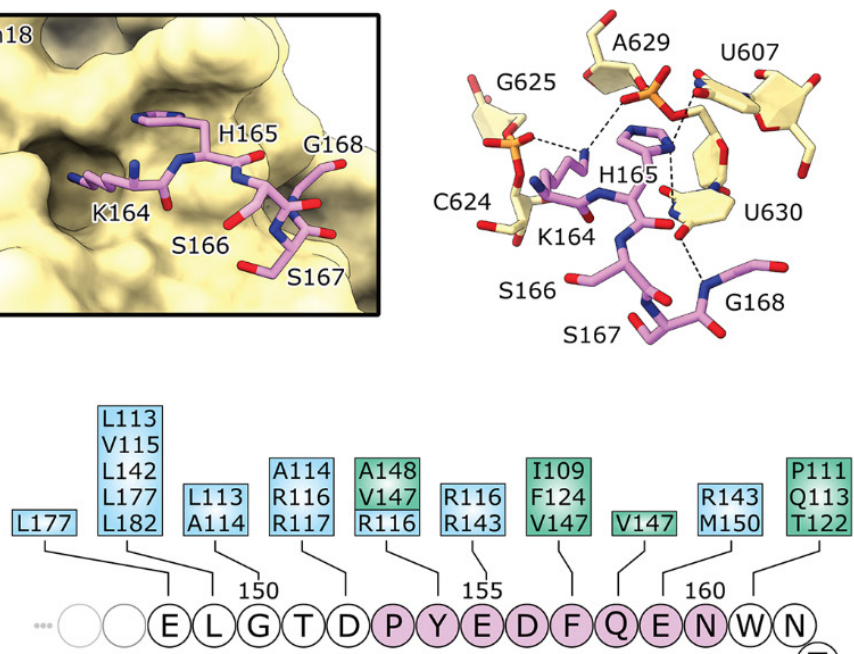

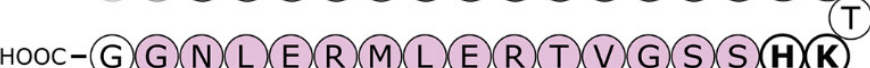
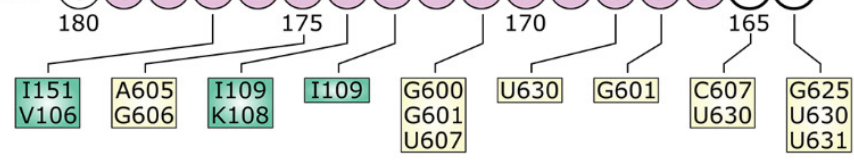

Fig. 3. Molecular basis of Nsp1 ribosome interaction and inhibition. (A) Cryo-EM map of in vitro reconstituted Nsp1-40S and segmented density of Nsp1-C, uS3 (97-153,168-189), uS5 (102-164) and rRNA helix h18 with the corresponding models; interacting residues are shown as sticks. (B) Nsp1-C surface, colored by electrostatic potential from -5 (red) to +5 (blue). (C) Model of Nsp1-C and surface representation of the models of uS3 (97-153,168-189), uS5 (102-164) and rRNA helix h18. Molecular interactions between Nsp1 and the ribosome. (D) mRNA entry channel, 40S head is removed. Nsp1C occupies the mRNA path [mRNA based on PDB-6YOG (47)]. (E) K164 and H165 of Nsp1 bind to a pocket on h18. (F) R171 and R175 of Nsp1 bind to the phosphate backbone of h18. (G) Negatively charged residues D152, E155 and E159 of $\alpha 1$ interact with uS3. (H) The hydrophobic interface of $\alpha 1$ and $\alpha 2$ binds to a hydrophobic patch on uS5. (I) Schematic summary of the interaction of Nsp1-C with uS3, uS5 and h18; residues belonging to $\alpha 1$ and $\alpha 2$ are colored in pink. 
A
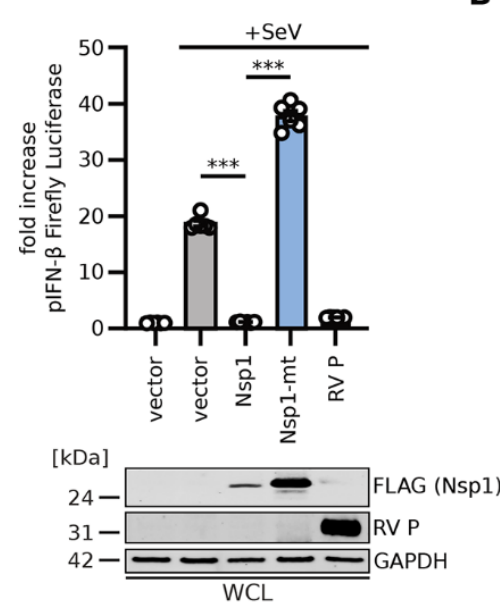

C

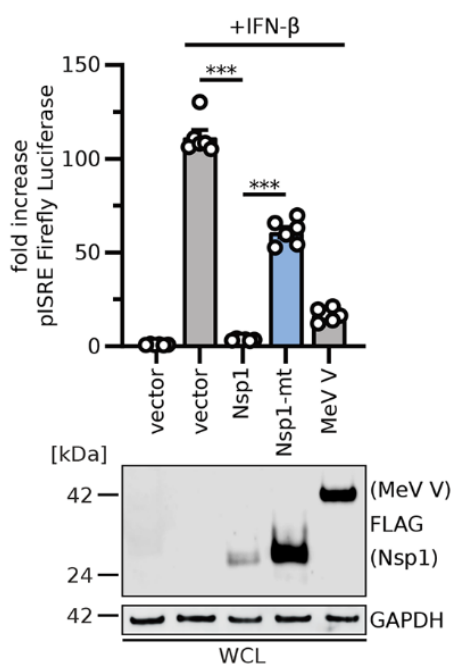

B
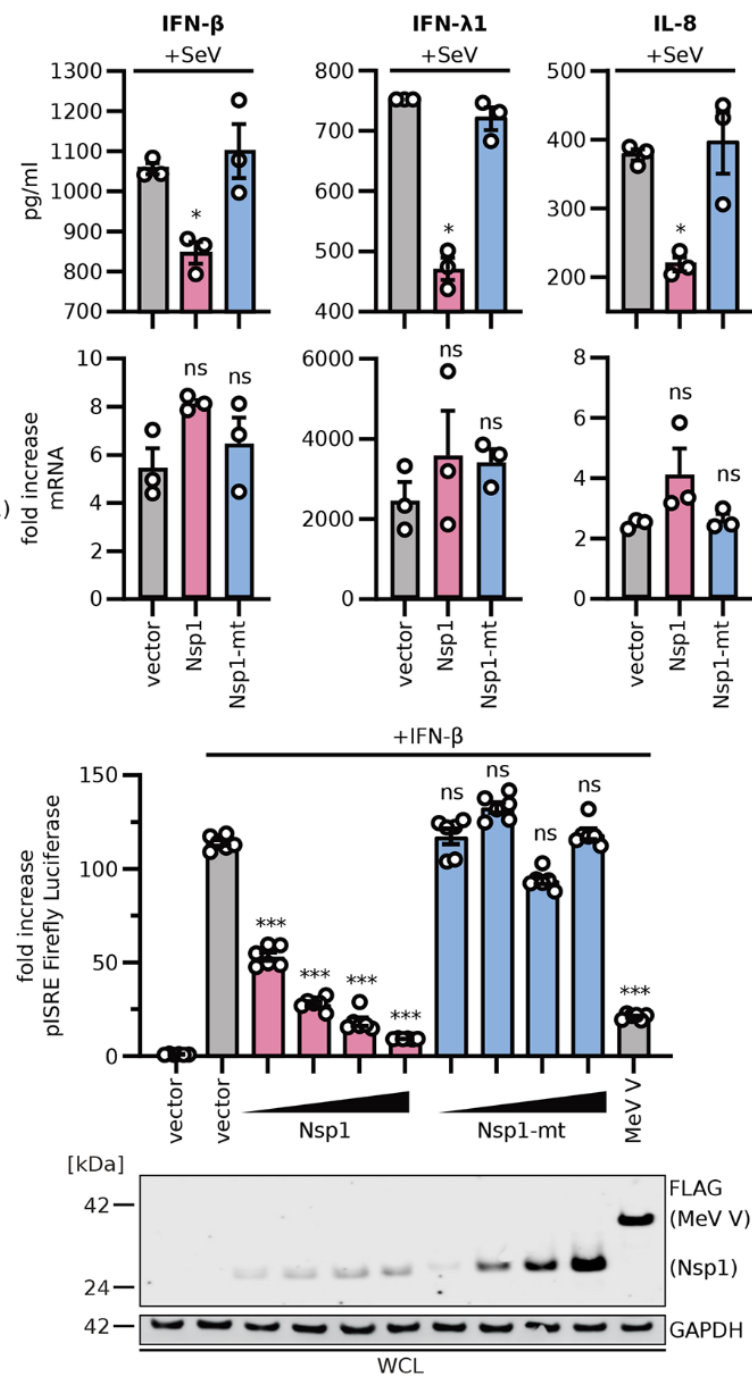

$\mathbf{E}$
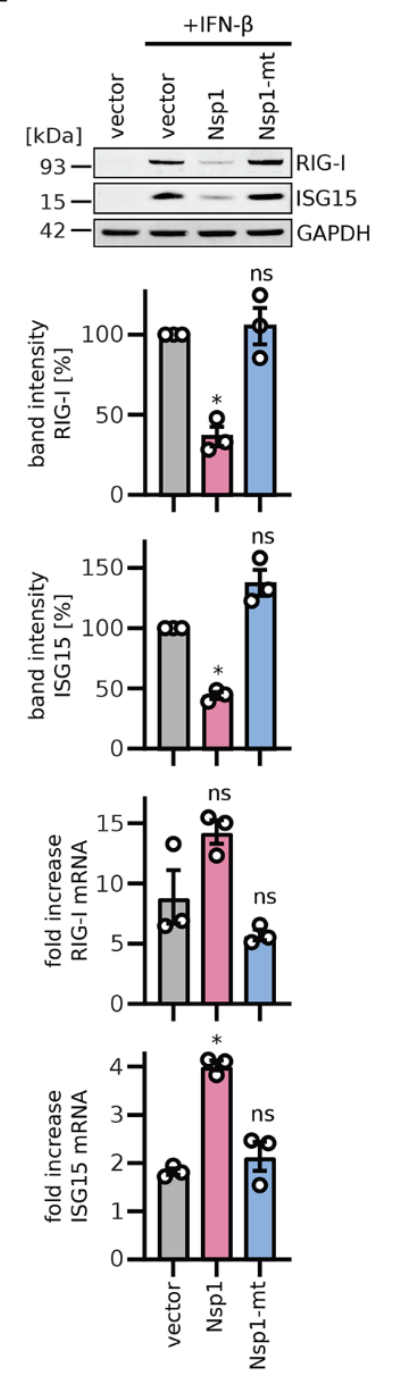

Fig. 4. Inhibition of the innate immune response by SARS-CoV-2 Nsp1. (A) Quantification of IFN$\beta$-promotor controlled firefly luciferase activity in HEK293T cells transiently expressing 3xFLAGtagged or non-tagged (RV P) proteins. Cells were infected with Sendai Virus (SeV) or left uninfected. Representative immunoblots of whole cell lysates (WCLs) stained with anti-RV P, anti-FLAG and antiGAPDH (bottom panel). (B) ELISA of IFN- $\beta$, IFN- $\lambda 1$ or IL-8 in the supernatant of HEK293T cells transiently expressing 3xFLAG-tagged proteins and infected with SeV (top panel) for 24 hours. qPCR of corresponding mRNAs (bottom panel). (C and D) Quantification of ISRE-promotor controlled firefly luciferase activity in HEK293T cells transiently expressing 3xFLAG-tagged proteins in single amounts (C) or increasing amounts (D) and treated with $1000 \mathrm{U} / \mathrm{mL}$ IFN- $\beta$ as indicated. Representative immunoblots of WCLs stained with anti-FLAG and anti-GAPDH (bottom panel). (E) Representative immunoblots and quantification of WCLs of HEK293T cells stimulated with 200 $\mathrm{U} / \mathrm{mL}$ IFN- $\beta$ stained for endogenous RIG-I, ISG15 and GAPDH. qPCR of the corresponding mRNAs (bottom two panels). In (A), (C), and (D), bars represent the mean of $n=6 \pm S E M$. In (B) and (E), bars represent the mean of $n=3 \pm S E M$. Unpaired student's $t$ test (Welch correction), ns, not significant; *, $p<0.01,{ }^{* *}, p<0.0001$. 